\title{
SISTEMA DE APOYO A LA TOMA DE DECISIONES PARA EL TELEMANDO DEL SISTEMA DE TRANSPORTE DE AGUA EN ALTA DE LA ACEQUIA REAL DEL JUCAR
}

\author{
Martinez Alzamora, Fernando (1) (P), Bou Soler, Vicente (2), Corts, Antonio (3), \\ Hervás, Alberto J. (4)
}

\begin{abstract}
${ }^{1}$ Cat. Ing. Hidráulica, Instituto de Ingeniería del Agua y M.A (IIAMA), Universitat Politècnica de València, Camino Vera, bq 5J, bajo 46022 Valencia, fmartine@hma.upv.es

2 Investigador contratado, Instituto de Ingeniería del Agua y M.A (IIAMA), Universitat Politècnica de València, Camino Vera, bq 5J, bajo 46022 Valencia, vibouso@dihma.upv.es

${ }^{3}$ Director de Operación. Operagua. Grupo Aguas de Valencia. acorts@aguasdevalencia.es

${ }^{4}$ Ing. Agrónomo. Director de Explotación. Unidad Sindical Usuarios del Júcar. usui@usuj.es
\end{abstract}

\section{Resumen}

La Acequia Real del Júcar (ARJ) es una de las infraestructuras de riego más antiguas de España, ordenada construir por Jaime I en 1258. Actualmente riega $20.000 \mathrm{Ha}$ de tierra fértil en la comarca de La Ribera, destinada en su mayoría a cítricos, aunque con un auge creciente del caqui. En su extremo final proporciona el agua necesaria para inundar los arrozales de la Albufera.

La Acequia Real puso en marcha en 2001 un ambicioso proyecto de presurización del sistema de riego y su conversión a goteo, destinado a mejorar de la eficiencia hidráulica del riego y la calidad de vida de los regantes, que ha conllevado un importante esfuerzo financiero por parte de las administraciones públicas.

Hasta el momento se ha completado la construcción de la red principal y de 10 de los 45 sectores de riego a goteo. El agua circula hoy día por una conducción a presión que aprovecha los $40 \mathrm{~m}$ de desnivel entre el túnel de la Escala de Tous y la zona regable, con el consiguiente ahorro energético.

La conducción principal da servicio a lo largo de sus $42 \mathrm{~km}$, a más de 100 puntos de descarga y a 21 tomas para el riego por goteo, con 253 válvulas motorizadas. También alimenta un bombeo de emergencia para suministrar agua a Valencia, by-paseando un tramo del canal Júcar-Turia. Para controlar todo el sistema por telemando se dispone de un potente SCADA ubicado en el Centro de Control de Benimodo (Valencia). Sobre él se ha instalado la aplicación SCARed, como Sistema de Apoyo a la Toma de Decisiones, el cual permite simular en tiempo real cualquier estrategia de riego, o la operación del bombeo de emergencia

\section{Abstract}

The Acequia Real del Jucar is one of the oldest irrigation infrastructures in Spain, ordered built by Jaime I in 1258. Currently irrigates 20,000 hectares of fertile land in the region of La Ribera, aimed mostly citrus, but with an increasing rise of persimmon. At the end provides enough water to flood the rice fields of the Albufera Lake.

The Acequia Real launched in 2001 an ambitious project to pressurize the transport system and transform the irrigation system to drip, in order to improve the hydraulic efficiency and the quality of life of the farmers, with the financial support of the public administration.

So far, the construction is completed in the main pipes and 10 of the 45 drip irrigation $\mathrm{s}$ sectors. The water carrier supply pressurized water taking advantage of the $40 \mathrm{~m}$ of head difference between the Escala tunnel at Tous dam and the irrigation area, with consequent energy savings.

Along its $42 \mathrm{~km}$, the main line serves over 100 discharge points and 21 connections to supply the drip irrigation network, with 253 motorized valves. It also feeds an emergency 
pumping to supply water to Valencia, bypassing a stretch of the Júcar-Turia canal. To control remotely the entire system there is installed a powerful SCADA system located in the Control Center of Benimodo (Valencia). Scared application works at the same place as a Decision Support System (DSS) fed from the SCADA data, which allows simulating in real time any irrigation strategy, or the operation of the emergency pumping

\section{1- Introducción}

La Acequia Real del Júcar (ARJ) es una de las infraestructuras de riego más antiguas de España, ordenada construir por Jaime I en el siglo XIII. Desde entonces ha sufrido muchas ampliaciones y mejoras, siendo la última de ellas el proyecto de modernización ejecutado en 2010 para transportar el agua a presión mediante de dos colectoras que recorren la margen derecha de la acequia a lo largo de $45 \mathrm{Km}$ de trazado, desde la toma en el azud de Antella hasta el término de Albal, donde la acequia actual descarga finalmente para alimentar los arrozales de la Albufera en la época de siembra. En su recorrido riega $20.000 \mathrm{Ha}$, en su mayoría de cítricos, pertenecientes a 21 términos municipales, actuando como elemento de vertebración de las comarcas de la Ribera Alta, Ribera Baja y Horta Sur.

En la figura 1 se muestra un esquema de la nueva instalación. Algunos datos significativos de la instalación son los siguientes: tubería doble en su mayor parte, con diámetros desde 2000 a 900 mm. Alimentación por gravedad desde el canal Júcar-Turia en un punto intermedio, con una carga máxima de $40 \mathrm{~m}$. Presión mínima de servicio en las tomas, $30 \mathrm{~m}$. Caudal máximo de transporte en presión, $10 \mathrm{~m} 3 / \mathrm{s}$. Número de puntos de descarga 101, agrupados en 21 tomas definitivas y otras 21 coyunturales que alimentan las fesas ubicadas en el origen de acequias para el riego a manta, aún operativas. La instalación dispone 253 válvulas motorizadas, 67 caudalímetros ultrasónicos y 75 sondas de presión, todos ellos telemedidos. A $20 \mathrm{Km}$ del punto de alimentación, se conecta una estación de bombeo de emergencia para reponer el agua en caso de avería del canal Júcar-Turia, que da servicio a la ciudad de Valencia, con una capacidad máxima de bombeo de 2,0 m³.

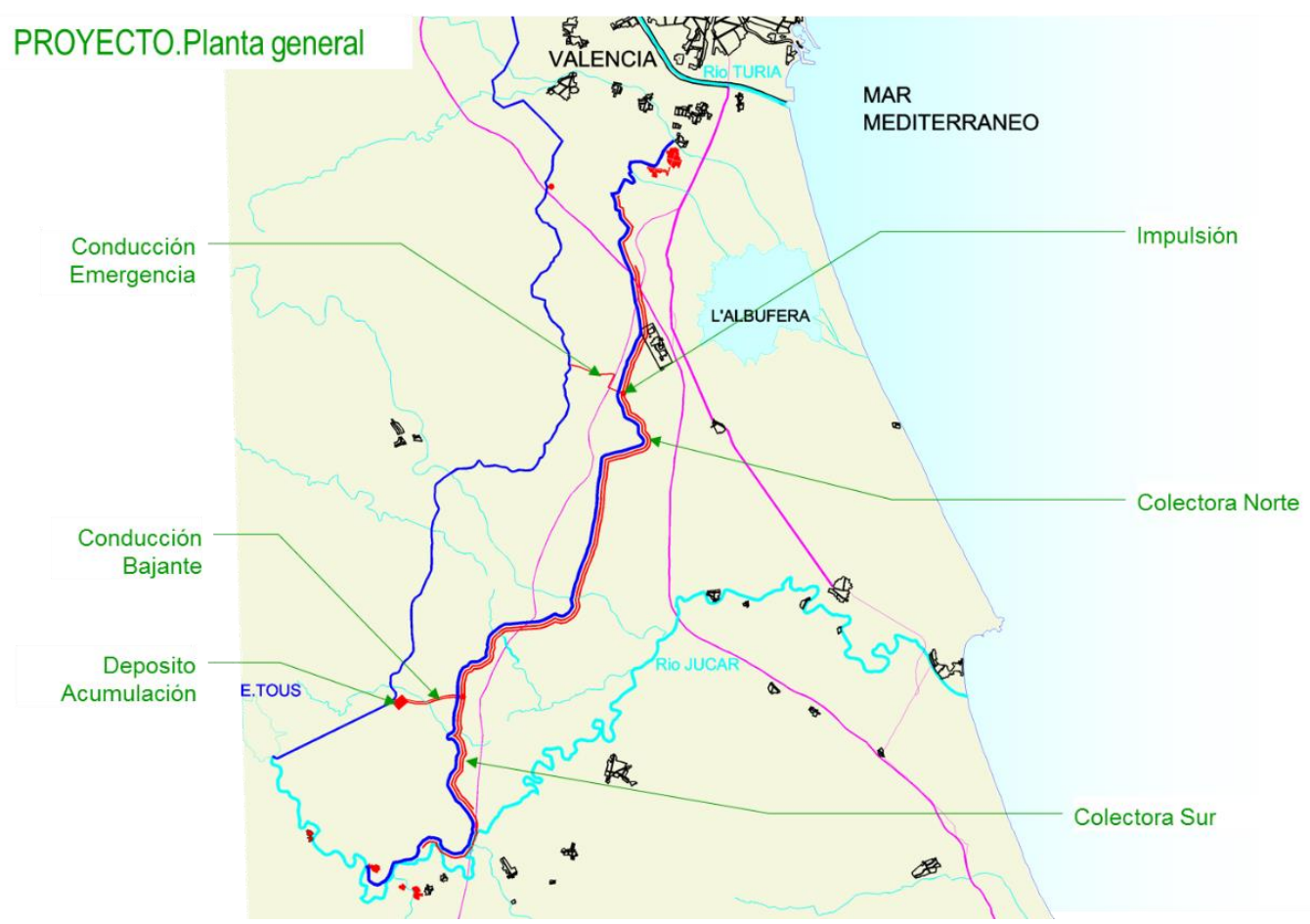

Figura 1. Esquema general de la instalación 
La magnitud de la instalación, el pequeño margen de pérdidas impuesto por la cota de alimentación, el posible crecimiento de la demanda en el futuro, las limitaciones de transporte en condiciones de demanda punta, y también en situaciones de emergencia, aconsejaban disponer de un Sistema de Apoyo a la Toma de Decisiones en tiempo real (DSS).

\section{2 - Materiales y métodos}

EI DSS de la ARJ está basado en la aplicación SCARed, desarrollada por el grupo REDHISP del IIAMA de la Universidad Politécnica de Valencia, la cual hace uso de un modelo hidráulico de la instalación construido sobre EPANET. SCARed es una aplicación de control en tiempo real para instalaciones hidráulicas, que aglutina diversos módulos orientados a:

i) capturar los valores de las variables hidráulicas desde diversas fuentes, como PLCs, SCADAs, data-loggers, ficheros planos, bases de datos, etc, bien en tiempo real o en tiempo diferido

ii) sincronizar, filtrar y almacenar la información de campo en una estructura de BdD propia

iii) mostrar los valores de las variables sobre un sinóptico dinámico creado con herramientas de desarrollo standard

iv) mostrar estos mismos valores sobre un fondo geográfico con la red superpuesta

v) consultar y comparar los valores de las variables elegidas en una ventana aparte capaz de alojar múltiples gráficos

vi) generar informes diversos, $y$

vii) proveer servicios al usuario a través de internet, como alarmas, sms, informes diarios, etc.

En la figura 2 se muestra el sinóptico general del sistema de transporte de agua en alta de la ARJ, tal como se muestra en la aplicación SCARed, el cual a su vez visualiza el comportamiento hidráulico del sistema.

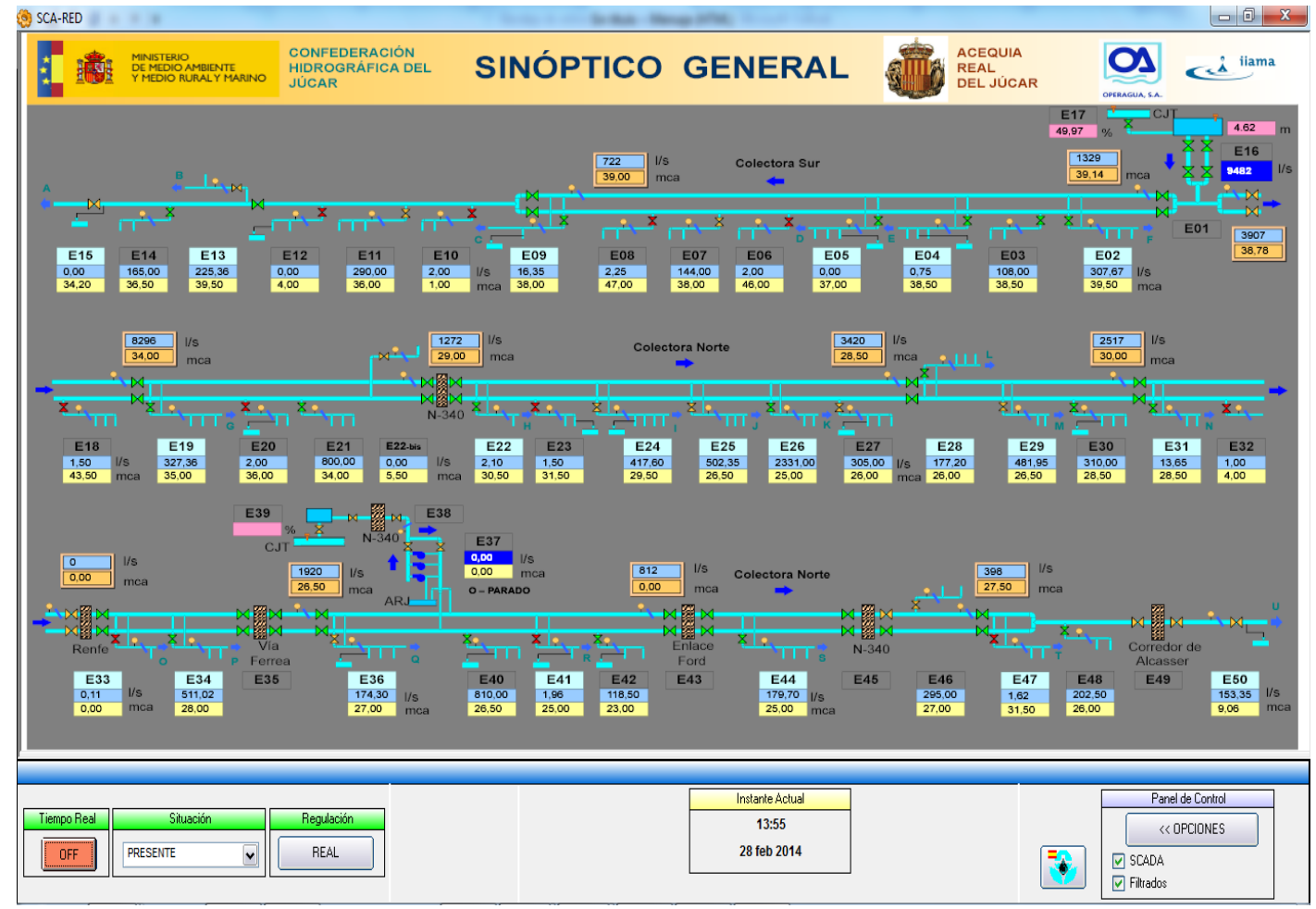

Figura 2. Sinóptico general de la ARJ en SCARed 
El sistema se alimenta por gravedad desde un depósito de cabecera a la cota $76 \mathrm{~m}$ (esquina superior derecha del sinóptico), el cual se abastece desde el canal Júcar-Turia. La tubería principal desciende duplicada desde el depósito hasta la margen derecha de la acequia, donde se bifurca en dos colectoras. La colectora Sur (primera línea del sinóptico), discurre a lo largo de 10,3 km en esa dirección, hasta morir cerca del azud de Antella, de donde parte la antigua Acequia Real; está duplicada en un $60 \%$ del recorrido. La colectora Norte (segunda y tercera líneas del sinóptico) discurre en sentido contrario, a lo largo de $32,1 \mathrm{~km}$, hasta el término de Alcasser, estando duplicada en casi el $90 \%$ del recorrido. Los diámetros de las conducciones oscilan desde los $2000 \mathrm{~mm}$ hasta los $900 \mathrm{~mm}$. A unos $22 \mathrm{~km}$ del punto de alimentación (tercera línea del sinóptico) se encuentra el bombeo de emergencia para suministrar agua a Valencia, el cual puede tomar el agua indistintamente de la colectora Norte o de la acequia, para descargarla de nuevo en el canal Júcar-Turia, lo que permite by-pasear el tramo del canal J-T entre ambos puntos.

Sobre el sinóptico general se han representado los cruces con otras servidumbres mediante rectángulos sombreados y junto a ellos las válvulas de seccionamiento que permiten aislar algunos tramos de tubería. Los puntos donde se ubican las válvulas están equipados con caudalímetros ultrasónicos y medidores de presión. A lo largo de los $42 \mathrm{~km}$ de conducción hay un total de 21 derivaciones coyunturales que permiten descargar el agua a las fesas (con una o varias tomas por derivación), y otras 21 derivaciones definitivas que, además de descargar también a las fesas, permiten que el agua continúe para alimentar el sistema de riego por goteo, compuesto por 45 sectores y dos o tres cabezales por sector. La instalación del riego por goteo es una de la mayores del país; suma un total 109 cabezales, 10.000 hidrantes y 50.000 electroválvulas, con sus correspondientes contadores. Existen además 15 puntos de descarga que retornan el agua a la Acequia Real en caso de necesidad. Todos los puntos de salida a la atmósfera están equipados por una válvula telemandada, que permite regular el uso del agua, según un programa de riego previamente establecido.

En cada una de las derivaciones hay instalada una estación remota, para el telemando de las diferentes válvulas. En la figura 3 se muestra el esquema detallado de una de ellas.

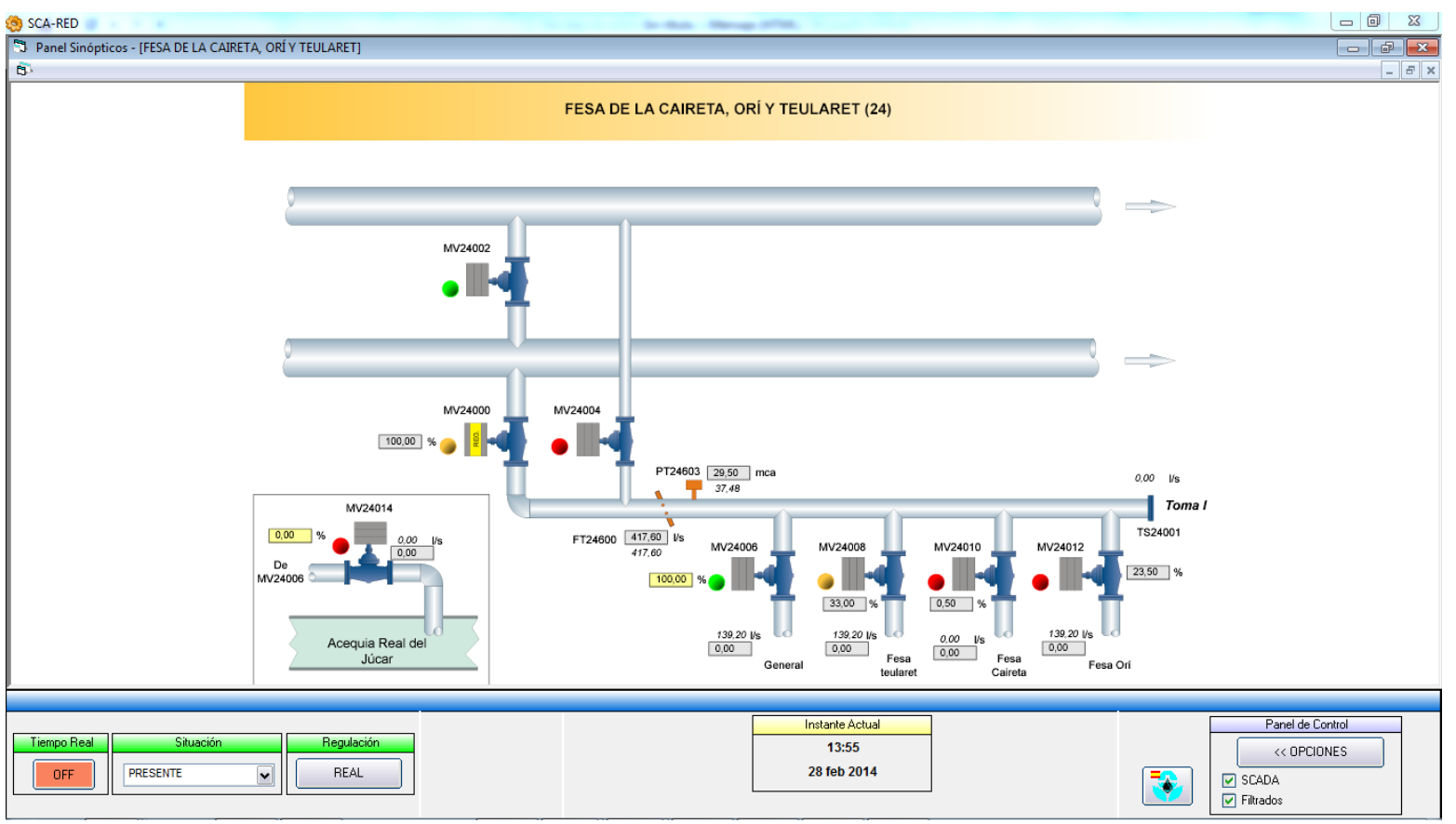

Figura 3. Sinóptico de una estación remota, con 4 descargas a fesas, una descarga a la Acequia, y una toma a la red de riego por goteo (toma I) 
En total se dispone de 50 estaciones remotas, con sus correspondientes sinópticos, todos ellos integrados en SCARed. A través de las remotas el SCADA central recibe señales del estado de todas las válvulas (señales MVxxxxx), del caudal total saliente de cada derivación, medido por un caudalímetro ultrasónico (señales FTxxxxx) y de la presión de suministro antes de las válvulas de descarga (señales PTxxxxx). Dicha presión es controlada por una válvula reductora de presión previa, cuya consigna está telemandada. La válvula reductora dispone además de un by-pass para poder alimentar directamente las descargas desde la otra tubería, y de una conexión entre los dos conductos, ambos controlados por su correspondiente válvula. Existen además algunas estaciones remotas especiales, como los cruces con servidumbres, las que controlan el sistema de alimentación y reparto del agua desde el depósito de cabecera, o la que controla el bombeo de emergencia.

Especial atención merece el sinóptico de la estación de bombeo de emergencia, el cual se muestra en la figura 4. En total se dispone de 3 bombas, todas ellas de velocidad variable. Las bombas pueden aspirar indistintamente de las colectoras o bien de la acequia, o incluso ambas opciones a la vez aislando para ello la aspiración de la primera bomba. Según el origen del agua, las bombas trabajarán a una u otra velocidad de giro, al cambiar la presión de aspiración. El caudal total impulsado puede consignarse a través del caudalímetro ubicado a la salida, y un automatismo se encarga de determinar las bombas activas y su velocidad de giro. También es posible el flujo inverso para retornar agua del canal a la acequia, o incluso alimentar las colectoras desde este punto, a cuyo fin se ha dispuesto la válvula de by-pass del extremo superior izquierdo.

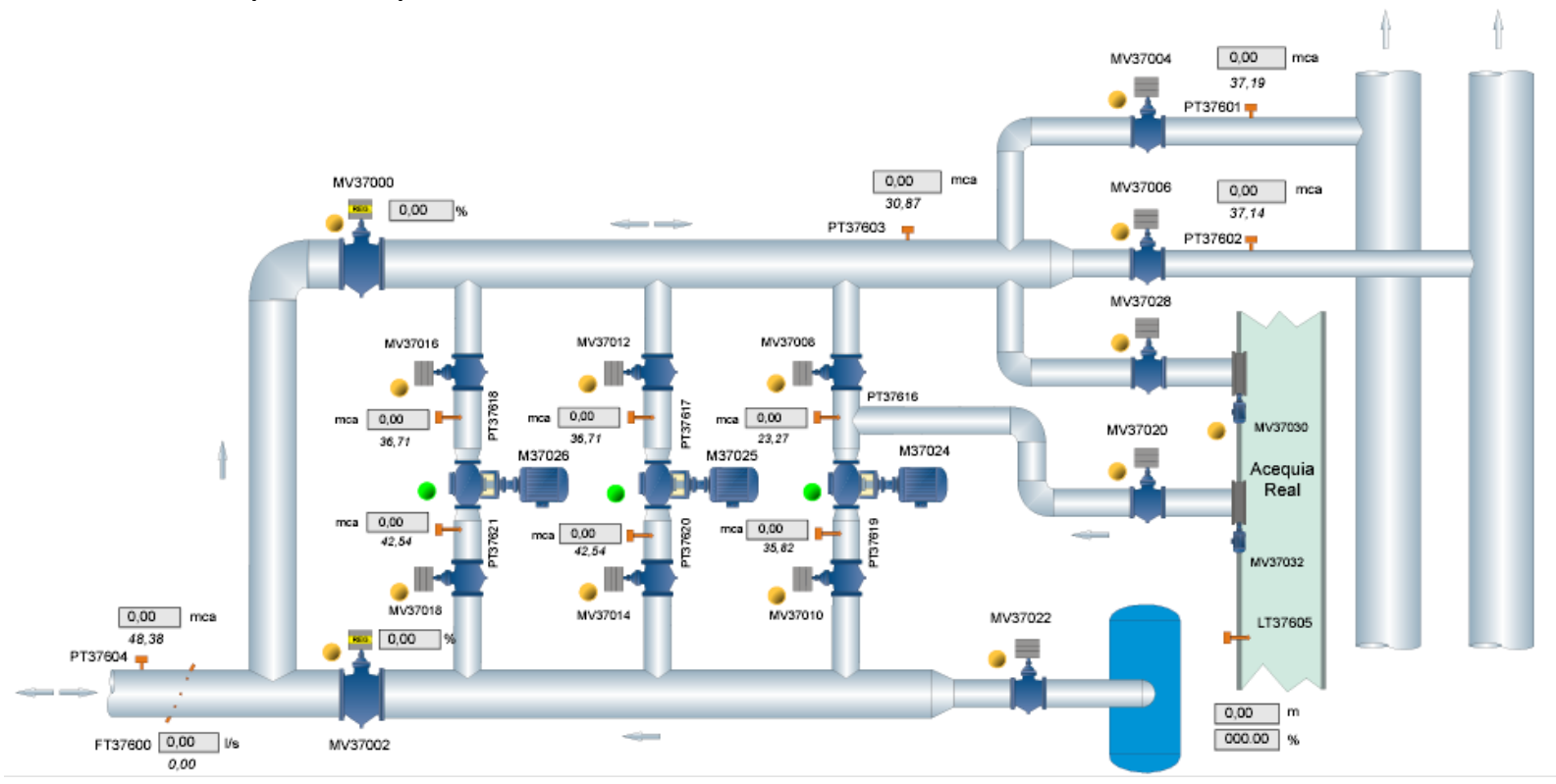

Figura 4. Sinóptico de la estación de bombeo de emergencia

Las señales de todas las magnitudes medidas desde cada estación remota se reflejan sobre su propio sinóptico. Además de ello, la presión regulada y el caudal total de cada derivación se muestran sobre el sinóptico general (figura 2), junto con los caudales totalizados en las válvulas de seccionamiento de las colectoras, lo que permite tener una visión de conjunto del comportamiento del sistema.

SCARed maneja en total 950 variables hidráulicas, procedentes de 253 válvulas motorizadas, 67 caudalímetros ultrasónicos y 75 sondas de presión, más algunas señales complementarias correspondientes a niveles en el depósito, posiciones de compuertas, estado de bombas, etc. A estas hay que añadir las variables calculadas y los caudales programados en las válvulas de descarga. 
SCARed lee o calcula continuamente toda esta información desde el sistema SCADA central, y la almacena en su propia base de datos, para posteriormente alimentar los diferentes sinópticos, así como el modelo hidráulico con el fin de llevar a cabo cualquier simulación. Los resultados de las simulaciones se muestran sobre los propios sinópticos de las estaciones, en una segunda casilla. Asimismo en una tercera casilla junto a los elementos de regulación regulables, se muestran los valores de las consignas aplicados en una determinada simulación.

El sistema SCADA central permite también regular por telemando el estado de cada válvula, ya sea su apertura o cierre, o bien su posición al objeto de alcanzar una determinada consigna de presión o de caudal de salida. La aplicación SCARed sin embargo, no está pensada para actuar directamente sobre las válvulas o bombas, tan solo para simular los resultados, actuando como un Sistema de Soporte a la Toma de Decisiones (SSD); solo el operador de la sala puede efectuar maniobras desde el SCADA de operación, o bien programarlas de acuerdo con las peticiones de los usuarios para el día siguiente.

\section{Modos de operación de SCARed}

SCARed puede operar de diversos modos: on line y off line, en presente, pasado o futuro, y en modo real o simulado. En el modo ON LINE simplemente muestra los valores de las variables medidas por el SCADA en tiempo real (presente), sobre los distintos sinópticos, con el valor añadido de poder mostrar también variables no telemedidas, pero calculadas por el modelo, como por ejemplo el caudal consumido en un sector o el caudal descargado a una fesa en particular. En este modo el valor de todas las variables es refrescado cada 10 segundos. Además, tras cada refresco, puede lanzarse el modelo hidráulico para reproducir la situación actual, y comprobar así constantemente la bondad del modelo.

En el modo OFF-LINE se dispone de tres opciones de consulta. El modo PRESENTE es el modo por defecto, y permite congelar la imagen para un análisis más detallado de la situación actual del sistema en modo real. Si se elige el modo simulado en presente, se pueden establecer nuevas consignas de operación (por ejemplo, abrir una toma nueva, cerrar un ramal, activar el bombeo de emergencia, etc) y simular la respuesta del sistema en la nueva situación. Las nuevas consignas de operación se establecen sobre unas pantallas que se abren al pinchar sobre el elemento de actuación, desde los sinópticos de las remotas. Los resultados se muestran sobre la segunda casilla de los sinópticos. Es una forma de anticiparse a una posible acción del operador, para conocer su efecto sobre la red antes de tomar una decisión. .

Estando en modo off-line, podemos seleccionar el modo PASADO, para reproducir escenarios pasados. En este caso hay que especificar el periodo de consulta entre una fecha inicial y otra final (por defecto se toman 24 horas), y pasar a observar a continuación el comportamiento real del sistema en cualquier instante de dicho periodo mediante una barra de navegación. Con ello el operador puede aprender sobre la respuesta del sistema ante diversos escenarios, o bien revisar las causas de un posible malfuncionamiento de la red en un momento determinado. Se pueden también contrastar los datos reales con los simulados por el modelo en cada instante, para proceder a su calibración si fuera necesario. En modo pasado es posible también permutar al modo simulado, y reproducir sobre la base de un escenario real, la respuesta del sistema ante unas condiciones de trabajo alternativas. Ello está concebido para el entrenamiento de operadores, por ejemplo, para analizar su capacidad de respuesta ante una situación de emergencia simulada. También puede utilizarse para verificar modos de operación alternativos más eficientes, por ejemplo, para mejorar las presiones de suministro en algunas derivaciones cambiando el programa de riegos. 
Pero la funcionalidad más importante de SCARed para el sistema en alta de la ARJ es su capacidad para simular situaciones futuras, y en particular el programa de riegos previsto para el día siguiente. En base a las necesidades hídricas de cada día, o a las demandas de los usuarios, se programa sobre una hoja Excel auxiliar la hora de apertura y cierre de las válvulas que descargan a las fesas o a la antigua acequia (figura 5), o bien se reproduce el programa de operación previsto en los cabezales del riego por goteo, si fuera el caso. A continuación SCARed leen todas las consignas y simula la operación del sistema para el día siguiente, pudiendo observando el operador las presiones resultantes, antes de aplicar definitivamente dicha programación. Pueden también simularse hipotéticas situaciones de emergencia que limitaran la capacidad de transporte en un momento determinado, o bien la operación simultánea del riego con el bombeo de emergencia. Además, las presiones de servicio dependerán del nivel del agua en la balsa de cabecera, cuya evolución durante la jornada de riego puede igualmente simularse.

\begin{tabular}{|c|c|c|c|c|c|c|c|c|c|c|}
\hline & & & & FECHA & $26 / 07 / 2013$ & & & & & \\
\hline & & & & & viernes, 26 de julio de 2013 & & & & & \\
\hline \multirow[t]{2}{*}{ Estación } & Salida & Inicio & Fin & $Q(1 / s)$ & Nombre estación & Cód Sinop. & $Q(\mathrm{~m} 3 / \mathrm{s})$ & $V(m 3)$ & \multicolumn{2}{|c|}{ Secc } \\
\hline & & & & & VERTIDOS A FESAS & & & & & \\
\hline 2 & NORTE & $7: 15$ & $18: 15$ & 30 & FESA ANGUILERA - MOTOR & MV02006 & 0,03 & 1188 & CC_CS & 2440 \\
\hline 2 & SUR & $1: 00$ & $1: 00$ & 0 & FESA ANGUILERA & MV02008 & 0 & 0 & & \\
\hline 2 & FESA & $7: 15$ & $18: 15$ & 250 & FESA ANGUILERA & MV02010 & 0,25 & 9900 & & \\
\hline 3 & FESA & $7: 15$ & $18: 15$ & 180 & FESA DE L'HORTA & MV03002 & 0,18 & 7128 & & \\
\hline 3 & GENERAL & $7: 15$ & $18: 15$ & 100 & FESA DE L'HORTA & MV03004 & 0,1 & 3960 & & \\
\hline 4 & FESA & $1: 00$ & $1: 00$ & 0 & ROLL DELS FRARES & MV04006 & 0 & 0 & & \\
\hline 4 & SUR & $1: 00$ & $1: 00$ & 0 & ROLL DELS FRARES & MV04008 & 0 & 0 & & \\
\hline 4 & NORTE & $7: 10$ & $18: 10$ & 180 & ROLL DELS FRARES & MV04010 & 0,18 & 7128 & & \\
\hline 5 & NORTE & $1: 00$ & $1: 00$ & 0 & ROLL DE MISSANA & MV05006 & 0 & 0 & & \\
\hline 5 & SUR & $1: 00$ & $1: 00$ & 0 & ROLL DE MISSANA & MV05008 & 0 & 0 & & \\
\hline 5 & FESA & $1: 00$ & $1: 00$ & 0 & ROLL DE MISSANA & MV05010 & 0 & 0 & & \\
\hline 6 & GENERAL & $1: 00$ & $1: 00$ & 0 & FESA CANALETA & MV06002 & 0 & 0 & & \\
\hline 6 & FESA & $1: 00$ & $1: 00$ & 0 & FESA CANALETA & MV06004 & 0 & 0 & & \\
\hline 7 & SUR & $1: 00$ & $1: 00$ & 0 & FESA ALMARIET - ALASQUER & MV07002 & 0 & 0 & & \\
\hline 7 & FESA & $1: 00$ & $1: 00$ & 0 & FESA ALMARIET - ALASQUER & MV07004 & 0 & 0 & & \\
\hline 8 & SUR & $1: 00$ & $1: 00$ & 0 & FESA NOVELLA & MV08002 & 0 & 0 & & \\
\hline 8 & FESA & $1: 00$ & $1: 00$ & 0 & FESA NOVELLA & MV08004 & 0 & 0 & & \\
\hline 9 & GENERAL & $0: 00$ & $23: 59$ & 20 & CRUCE TOUS-ALBERIQUE & MV09006 & 0,02 & 1726,8 & S_E9 & 1700 \\
\hline
\end{tabular}

Figura 5. Programación de riegos para el día siguiente (extracto)

SCARed cuenta además con herramientas complementarias para efectuar análisis sobre valores históricos de cualquier señal en un periodo determinado, permitiendo su comparación con la misma señal en otros periodos o con otras señales para el mismo periodo. En la figura 6 se muestra como ejemplo el caudal total horario suministrado a lo largo de 2013. En el panel de la izquierda se selecciona la señal y el periodo con diversas opciones, entre las que destaca la posibilidad de combinar señales. A la derecha se muestras los resultados, apilados por pestañas. 


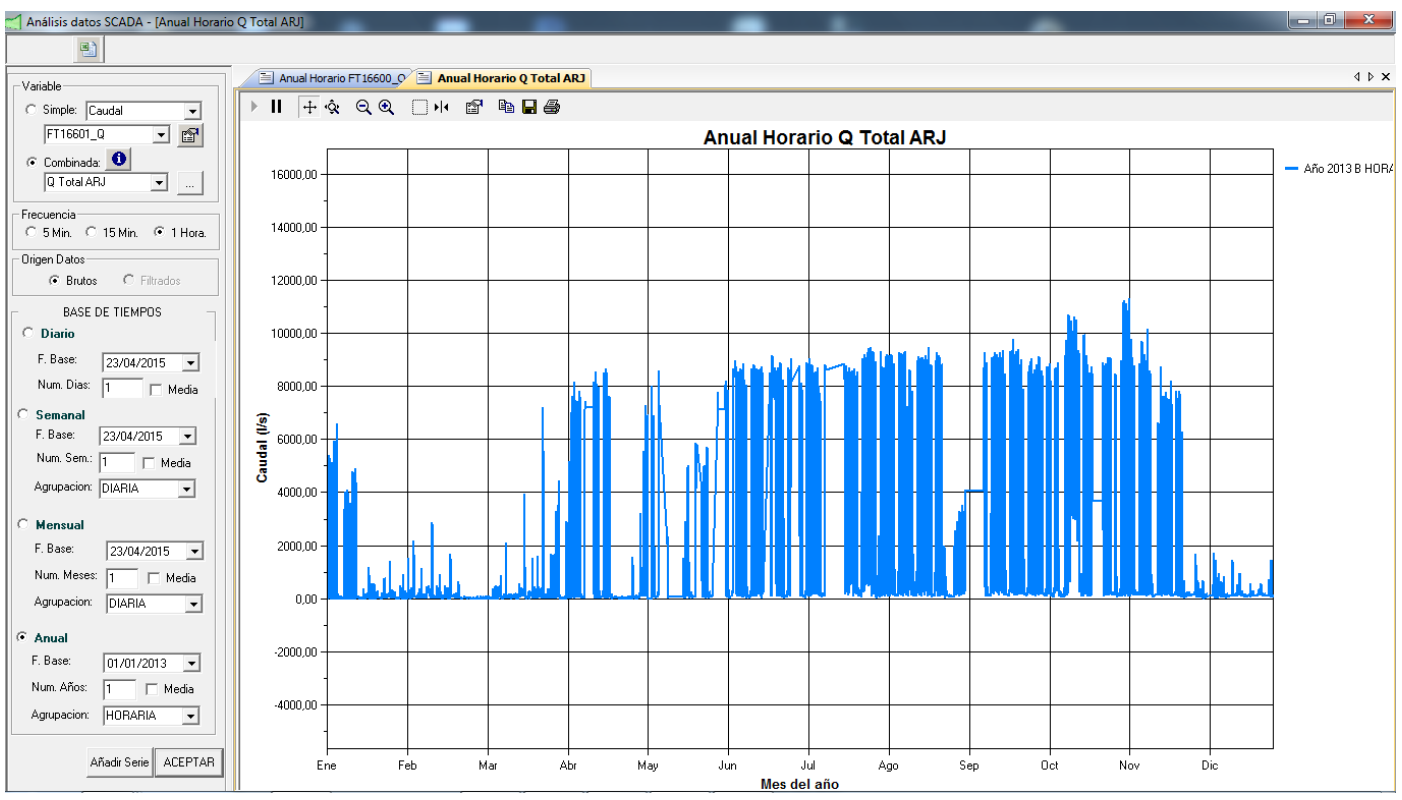

Figura 6. Caudal horario total suministrado en 2013

\section{Confección del modelo hidráulico}

La construcción del modelo matemático con EPANET ha supuesto un reto, para combinar las especificaciones de la demanda del goteo a través de sus curvas de modulación con la apertura y cierre de válvulas a determinadas horas mediante leyes de control. Las tomas para el goteo,se han modelado como nudos de demanda con un caudal base uniario. Los factores de modulación salen de combinar los programas de riego establecidos para todos los cabezales alimentados por cada toma. Las válvulas de descarga, por su parte, se han modelado como emisores provistos de una válvula limitadora de caudal, ya que el caudal descargado a las fesas es consignable, y depende del grado de apertura de las válvulas. Dado que todas las derivaciones van provistas de una válvula reductora de presión previa, el coeficiente de pérdidas del emisor de cada toma se ha determinado para permitir la descarga del caudal límite a válvula abierta, para una presión de consigna de la reductora de $25 \mathrm{~m}$. De este modo, el operador puede fijar la hora de apertura y cierre y el caudal programado para cada válvula de descarga (figura 5), lo que se simula mediante leyes de control temporales.

La simulación de los diferentes modos de trabajo de las bombas de emergencia se ha llevado a cabo mediante reglas complejas, ya que la puesta en marcha y parada de cada modo lleva asociada la apertura y cierre de varias válvulas. El caudal bombeado es igualmente consignable, lo que se ha simulado nuevamente con una válvula limitadora de caudal a la salida. El número de bombas activas y su velocidad de giro en función del caudal demandado se ha modelado igualmente mediante reglas complejas.

Finalmente la entrada de caudal desde la toma hasta el depósito de cabecera se ha simulado mediante una válvula limitadora de caudal, cuyo caudal de consigna se ha hecho depender del grado de apertura de las compuertas, lo que permite simular las variaciones de nivel en el depósito en función de los caudales entrante y saliente cada hora.

En la figura 7 se muestran los resultados de una simulación sobre EPANET, los cuales pueden obtenerse en cualquier momento al pulsar el icono situado en la barra inferior. De este modo, el usuario puede realizar cualquier simulación adicional trabajando directamente sobre el entorno de EPANET, o bien consultar otros resultados sobre dicho entorno. 


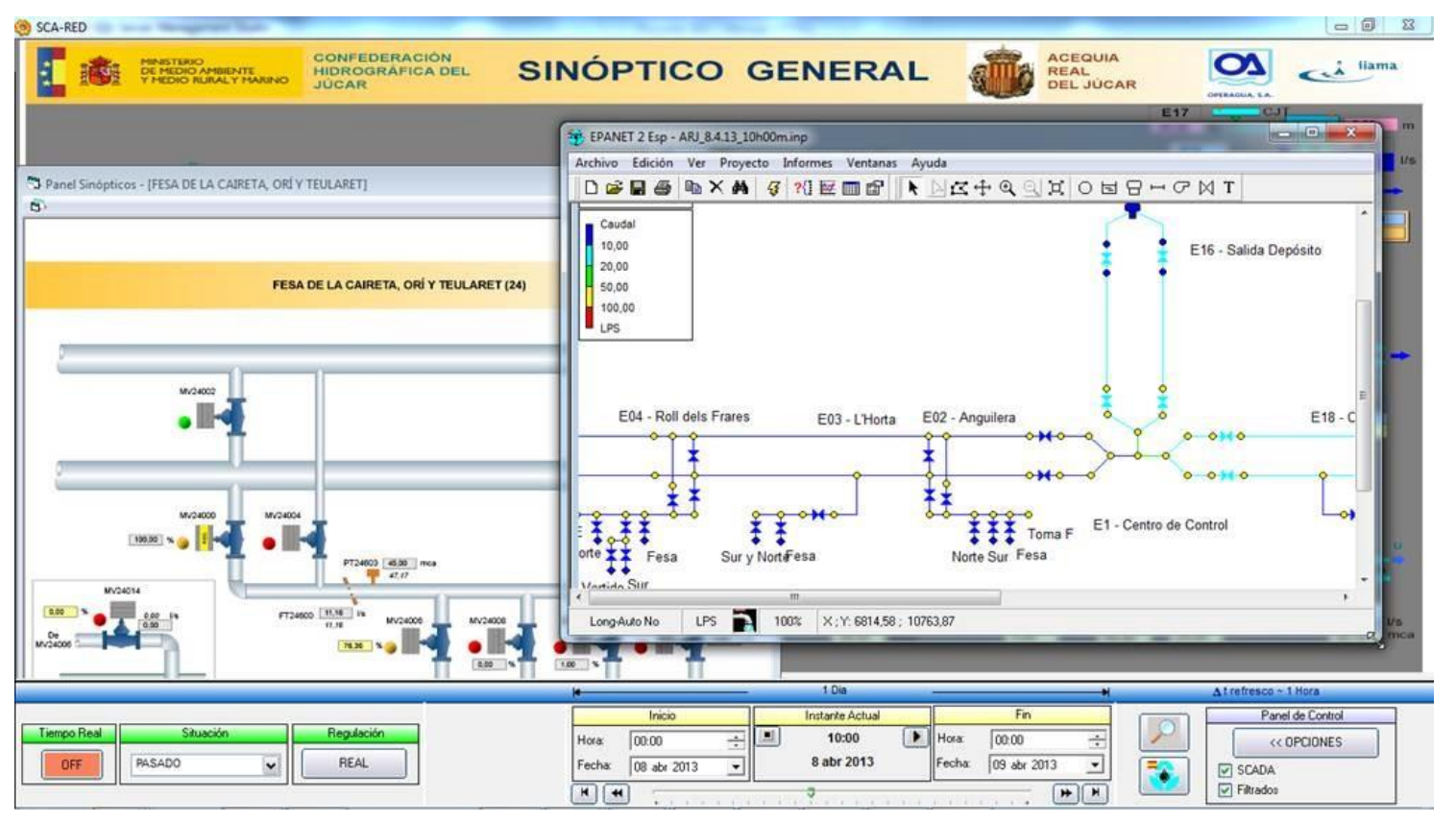

Figura 7. Exportación del modelo directamente al entorno de EPANET

\section{3 - Resultados}

La aplicación SCARED se puso en marcha en Octubre 2010, en el Centro de Control de Benimodo, tras un periodo de pruebas durante el cual se coordinó su funcionamiento con el SCADA de operación, un sistema Vijeo Citect de Schneider, instalado por la empresa Tefralux. Desde entonces la garantía en el funcionamiento de la aplicación está en manos de la empresa Operagua, del grupo Aguas de Valencia, la cual se encarga también del mantenimiento electromecánico y la reparación de las tuberías y valvulería.

SCARed ha funcionado sin problemas desde entonces. El volumen de datos almacenado en estos 5 años ha permitido tener un conocimiento exhaustivo del comportamiento del sistema, bajo las múltiples circunstancias acaecidas en este tiempo. La ARJ utiliza la aplicación para verificar la viabilidad del programa de riegos establecido diariamente, el cual va cambiando con la época del año, y también a medida que se van incorporando nuevos sectores de riego por goteo. El bombeo de emergencia, afortunadamente, no ha necesitado ser activado hasta ahora, aunque está en previsión utilizarlo mientras se efectúan algunas reparaciones en el canal J-T.

\section{4 - Conclusiones}

La conexión en tiempo real de un modelo hidráulico con el SCADA de operación, constituye una poderosa herramienta para la toma de decisiones. Además de permitir anticiparse a cualquier acción, permite también revisar situaciones pasadas, o en este caso, analizar la viabilidad del programa de riegos establecido para el día siguiente, a fin de garantizar las presiones de suministro. En otras instalaciones provistas con sistemas de bombeo, puede utilizarse para optimizar el programa de riegos, a fin de reducir los costes de operación.

Para la confeccionar y ejecutar el modelo hidráulico utiliza un simulador estándar como es EPANET, con el cual es posible simular tanto las demandas de riego mediante curvas de modulación, como la descarga directa de las válvulas a la atmósfera, estableciendo la hora de apertura y cierre de las mismas, siendo en este caso el caudal fijo o dependiente de la 
presión. Otras leyes de operación más sofisticadas, propias de las estaciones de bombeo, pueden simularse igualmente utilizando reglas de control simples o complejas.

SCARed emula los sinópticos utilizados en los SCADAs reales, para facilitar su uso por parte de los operadores, y es compatible con cualquier SCADA, siempre que se establezca un protocolo de comunicación adecuado, ya que gestiona su propia base de datos. Además de la ARJ, SCARed se encuentra también operativo en otros dos grandes sistemas de abastecimiento, como son Valencia y Málaga. En el futuro, se pueden desarrollar nuevas funcionalidades basadas en el uso de modelos, para cubrir las necesidades particulares de cada usuario.

\section{5 - Agradecimientos}

Los autores agradecen a la ARJ por su firme apuesta en la implantación de nuevas tecnologías orientadas al uso eficiente del agua, en una infraestructura de tan gran envergadura, contribuyendo así a los objetivos establecidos del Plan Hidrológico Nacional. Asimismo los autores de la aplicación agradecen la confianza de la empresa Operagua por hacer de vehículo conductor y la colaboración de los técnicos de Tefralux para facilitar la comunicación de SCARed con el SCADA de operación.

\section{6 - Bibliografía}

Acequia Real del Júcar. (2013). Página web. http://www.acequiarj.es/

Alonso, J.M., Alvarruiz, F., Guerrero, D., Hernández, V., Llopis J.D., Ramos, E., Martínez, F., Bou, V. Bartolín, H. (2004). Simulation of control strategies in water distribution systems, using SCADA in conjunction with calibrated models obtained from GIS. Proc. 6th Int. Conf. Hydroinformatics, Singapur. World Scientific Pub. Co.

Bou, V., Martínez, F., Conejos, P. (2006). SCA-Red, a general purpose SCADA application for taking decisions in real time with the aid of a hydraulic model. 8th Annual Water Distribution Systems Analysis Symposium. WDSA. Cincinnati (Ohio)

Hervás Ferrer, A.J. (2009). La modernización de la Acequia Real del Júcar. II Jornadas sobre la Gestión Eficiente del Riego. Univ. Politécnica de Valencia. 14-15 Dicbre 2009

Ingedul, P. (2007). Real-Time Forecasting with EPANET. World Environmental and Water Resources Congress ASCE

Janke, R. J., Haxton, T. M., Boccelli, D., Kramer,A., Matracia, A., Panguluri, S., Uber, J. and Hachett, S. (2010). How Accurate Is A Hydraulic Model? U.S. EPA, Office of R\&D, Nat. Homeland Security Center. Water Infr. Prot. Division

Martínez, F., Hernández, V., Bartolín, H., Bou, V., Alvarruiz,F., Alonso, J.M. (2005). CALNET project: building and updating water distribution models from GIS+CIS+O\&M+SCADA . CCWI 2005 , Exeter, UK. Water Management for the 21st Century, Vol. One, pp 209214.

Martínez, F., Hernández, V., Alonso, J.M., Rao, Z., Alvisi, S. (2007). Optimizing the operation of the Valencia water-distribution network. Journal of Hydroinformatics, Vol 9, no 1, pp $65-78$

Martinez Alzamora, F, Bou Soler, V, Conejos Fuertes, P. Díaz Martínez, J. (2013) Conexión SCADA-modelo para la simulación en tiempo real del sistema de abastecimiento de 
agua a Valencia y su área metropolitana. III Congreso Ingeniería del Agua. Univ. Politécnica de Valencia. Actas JIA Vol 2. Octubre 2013 pp

Menold, P.H., Pearson, R.K., Allgöwer, F. (1999). Online outlier detection and removal. Proceedings of the 7th Mediterranean Conference on Control and Automation (MED99) Haifa, Israel

OPC Foundation. (2001). OPC Data Access Interface Specification 2.05

Quevedo, J., Puig, V., Cembrano, G., Blanch, J., Aguilar, J., Saporta, D., Benito, G., Hedo, M., Molina, A.. (2010). Validation and reconstruction of flow meter data in the Barcelona water distribution network. Control Engineering Practice 18, pp 640-651

Rofail, A., Shohouf Y. (1999). Mastering COM and COM+. Ed. Sybex

Rossman, L. (2000), Epanet 2 User's Manual. Environmental Protection Agency. Cincinnati, $\mathrm{OH}, \mathrm{USA}$.

Salas, P., Domene, J., Martinez, F., Bartolín, H., Bou, V. (2010). La gestión informatizada del agua inyectada y registrada en la red sectorizada de Málaga, para la mejora de su rendimiento. XXX Jornadas Técnicas AEAS. Sevilla.

SCARed (2010) Software para la simulación en tiempo real de estrategias de control en Redes de Distribución de Agua. Grupo REDHISP. IIAMA. Univ. Politécnica de Valencia. http://www.iiama.upv.es/iiama/src/ficha software.php?lang=\&id=4

Scheneider Electric. (2013) Viejo Citect. Quick start tutorial. http://www.schneiderelectric.com/products/ww/en/5100-software/5135-operating-monitoring/1500struxurewarescada-expert-vijeo-citect/

Velickov, S.. (2012). Improving efficiency of water utilities: real-time modelling and Scada data assimilation. Smart Water Network Conference. Utrech

Tiburce V., Chopard P., Hamon J., Elain C. and Green A. (1999). Linking SCADA to a Hydraulic and Water Quality Simulator at the 'Centre des Mouvements de l'Eau' (CME) in Paris. CCWI'99 Conf, Exeter, UK, Water industry systems: modeling and optimization applicat. Vol. 1, pp 243-257.

Wells, G. (2001). Code-Centric T-SQL Programming with Stored Procedures and Triggers, APress. 